\title{
COMMUNICATIONS
}

\section{EXPERIMENTAL OCCLUSION OF RETINAL ARTERIOLES* (USING GRADED GLASS BALLOTINI)}

BY

\author{
NORMAN ASHTON AND PAUL HENKIND $\dagger$ \\ From the Department of Pathology, Institute of Ophthalmology, University of London
}

DISEASE of the retinal vessels usually involves in varying degree the whole retinal vascular system, so that in considering the pathogenesis of any particular lesion it is often difficult to decide whether it is primarily related to the arterioles, capillaries, or venules. From the experimental point of view it would be of value if the sequelae of uncomplicated lesions in each part of the vascular tree could be separately studied. With this object in mind we have sought, as an initial step, a suitable material with which to occlude in vivo the retinal arterioles. The artificial emboli must, of course, be the appropriate size, and should be inert, easily identifiable, and simple to prepare, to inject, and to store. We have found that small glass balls or ballotini are eminently satisfactory for this purpose and, to make the method available to others pursuing similar lines of work, we report in this preliminary communication a description of our technique together with a brief survey of some of our findings.

\section{Material and Methods}

(a) Ballotini $\ddagger$.-These are lead glass spheres ranging in diameter from approximately 15 to $75 \mu$. They can be graded using nylon gauze $\S$ as a sieve to provide concentrations of any required diameter. We have generally used a mixed suspension containing ballotini 15 to $40 \mu$ in diameter.

(b) Suspending Media.-Supplied as a whitish powder the ballotini must be suspended in a suitable medium before use, and a sterile solution of gum arabic has been used for this purpose. A minute quantity of ballotini, enough to cover the tip of a small spatula, is added to $10 \mathrm{ml}$. of the medium. The balls slowly sink and can be resuspended by vigorous agitation.

(c) Injection of Ballotini.-The suspended ballotini may be injected with an ordinary syringe provided that both the barrel and plunger are well lubricated with either Vaseline or silicone grease. If this precaution is not observed the ballotini will lodge between the plunger and barrel, jamming the unit. Flexible tubing and a plastic cannula (Braunula)\|

\footnotetext{
* Received for publication December 30, 1964 . Neurological Diseases and Blindness of the National Institute of Health.

$\ddagger$ Kindly supplied by the English Glass Co. Ltd., Leicester, England.

$\S$ Supplied by the Zurich Batting Cloth Mfg. Co. Ltd.

II Braunula-available from Armour Pharmaceutical Co. Ltd., Eastbourne, Sussex, England.

17 
complete the injection apparatus. During the injection the syringe should be gently agitated to prevent the ballotini from settling.

(d) Operative Technique.-The bulk of the present work was carried out using either kittens or adult cats, but similar successful injections have been carried out in monkeys, pigs, and rats. The cats were anaesthetized with intraperitoneal sodium pentobarbitone (Nembutal) and their pupils were dilated with 1 per cent. atropine drops. Either the left or right common carotid artery was isolated through a vertical paracentral incision in the neck. The artery was gently separated from the adjacent structures, particularly the vagus nerve, and a ligature was tightly tied at its exposed proximal end. Ophthalmoscopic examination performed during and immediately after ligation revealed no visible change in either the ipsilateral or contralateral retinal vessels. A loose ligature was then placed around the exposed distal end of the artery, and a cannula was inserted into the vessel. In every instance there was a backflow of arterial blood into the cannula and immediately thereafter 1-8 ml. of ballotini suspension were injected into the carotid artery. During injection the ipsilateral fundus was examined by direct ophthalmoscopy. After the injection the cannula was removed and the distal carotid ligature was firmly tied. (Ligature alone was shown not to affect the retinae of the animals used.) The skin incision was closed with silk sutures, Aureomycin applied to the wound site, and a protective dressing placed around the neck.

(e) Follow-up.-Animals surviving operation were examined daily for physical and ocular abnormalities and fundus photographs or paintings were made at appropriate intervals. The cats were examined without sedation during the entire post-operative period.

\section{Findings}

More than 40 cats have been studied to date and approximately two-thirds of them have been successfully injected with ballotini. Over one-half of those animals with retinal arteriolar ballotini have survived the immediate post-operative period, survival occurring more frequently in cats receiving less than $3 \mathrm{ml}$. of the suspension. Noteworthy was the apparent docility of a number of the surviving animals-presumably due to the effect of ballotini in the cerebral vessels, but no other neurological abnormalities were observed.

The clinical ocular findings in successfully injected cats were primarily localized to the fundus. Indeed, there was evidence of involvement of the anterior segment in only one animal, in which a plastic exudate was noted in the right anterior chamber two days after injection of ballotini into the right carotid artery. The fundus findings can be divided clinically into two groups:

(1) Vascular lesions - that is, specific retinal vascular changes, including (a) visible ballotini in the arterioles; (b) sludging phenomena in the blocked arterioles and adjacent venules; (c) arteriolar anastomoses; (d) venous anastomoses; and (e) retinal neovascularization (only in the immature retina).

(2) Extravascular lesions (but obviously vessel dependent)-including (a) retinal oedema; (b) "cotton-wool" patches; (c) retinal haemorrhages; and (d) retinal atrophy and cystic degeneration.

In the majority of successful experiments ballotini were visible immediately after injection, generally at arteriolar bifurcations and particularly over the tapetal (upper half) of the fundus. On several occasions corneal oedema at the time of operation hindered ophthalmoscopy, but subsequent examinations revealed the intra-arteriolar ballotini, which usually appeared as black dots with a glistening light reflex (Fig. 1). Often, directly after an arteriole was blocked, vascular sludging or "cattle trucking" 


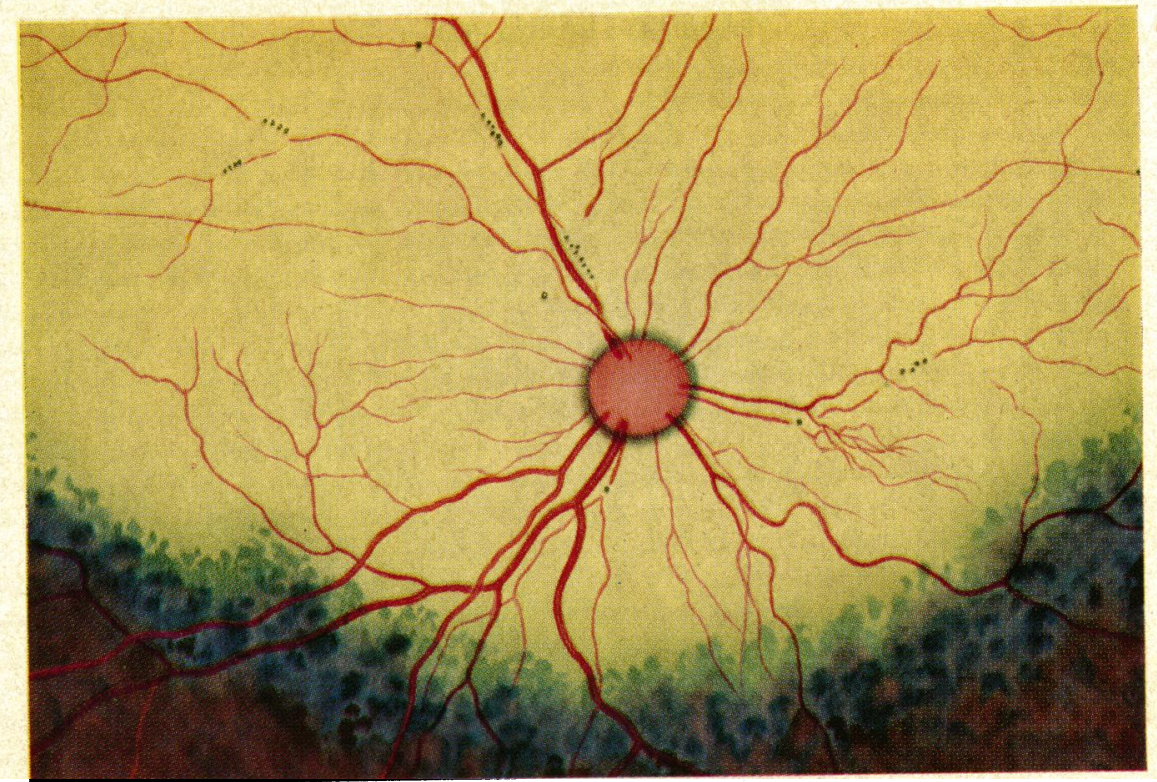

FIG. 1.-Cat 1, right eye. Ballotini seen in retinal arteries several days after injection.

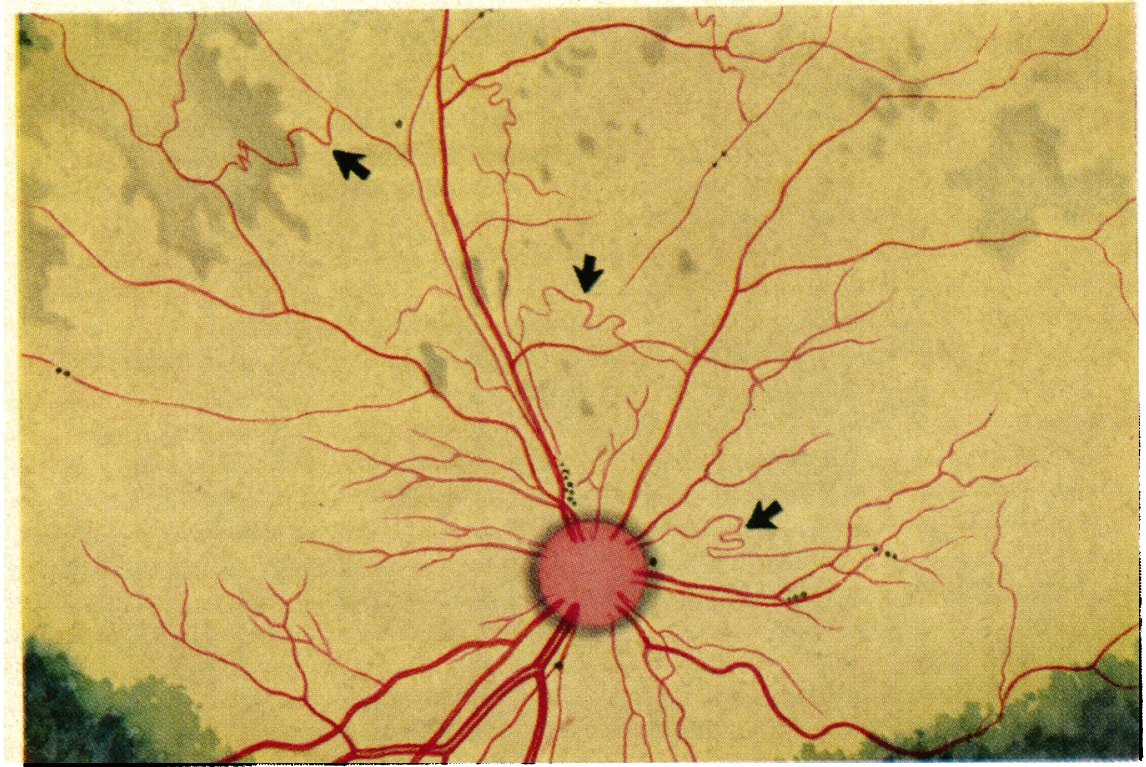

FIG. 2.-Cat 1, right eye. Fundus painting showing development of arterial anastomoses (arrows) four weeks after injection of ballotini. Note grey areas of "geographic" retinal atrophy. 
became evident distal to the block. Only rarely was there obvious narrowing of the blocked vessel at the time of operation, but the affected vessels narrowed and often became obliterated in a period of days or weeks. Not infrequently a retrograde flow was seen in the artery within minutes of ballotini block, and in this process small clumps of blood were seen to travel centripetally in the arteriole towards the disc and to leave the main channel via a smaller branch vessel lying distal to the block. Sludging of venous flow draining the neighbourhood of blocked arterioles was a prominent feature in a number of animals. Sludged flow (both arteriolar and venous) persisted for several days in a few animals - after which time the blood column appeared continuous and it was then impossible to determine the direction in which the blood was moving. Anastomoses between blocked and adjacent patent arterioles were commonly noted in this study. Visible anastomoses first appeared several days after occlusion of an arteriolar branch and continued to enlarge until they attained a rather impressive size, almost as large as that of the blocked arteriole itself (Fig. 2). These

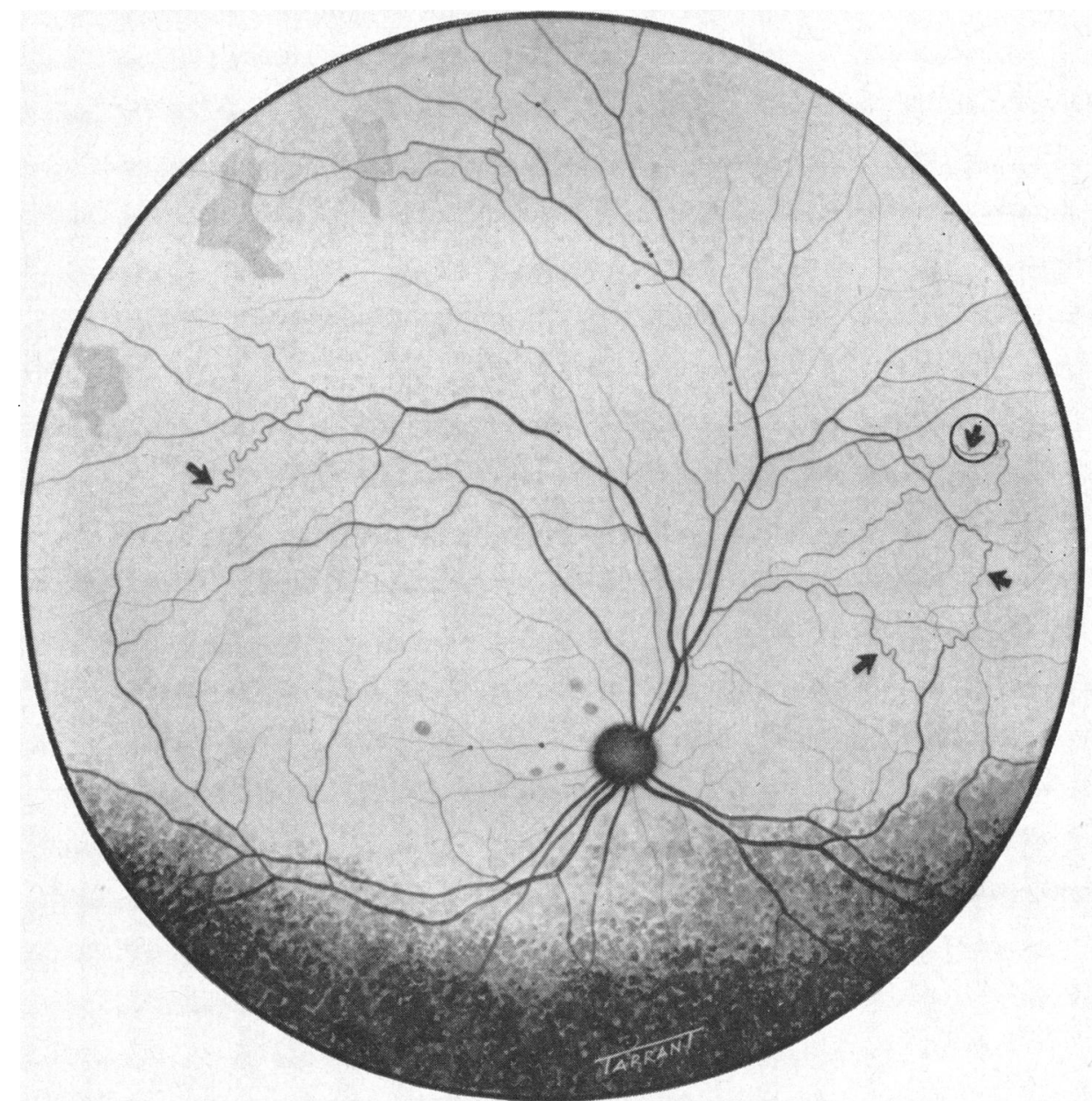

FIG. 3.-Cat 28, right eye. Venous anastomoses (arrows) ten days after ballotini injection. Circled arrow indicates an arterial anastomosis. 
vascular communications appeared to be enlarged interarteriolar capillary channels rather than new-formed vessels. Similar venous anastomoses occurred in several animals, always in the vicinity of blocked arterioles, and always subsequent to sludging, which had been noted both in the retinal arterioles and venules (Fig. 3). True neovascularization was never obtained in the adult animal but intravitreal capillary proliferations were induced in a kitten when the ballotini were introduced at a stage of immature retinal development, namely at eight days of age. The proliferation appeared in the retinal periphery arising from the venous side of the circulation as the arterioles were blocked by ballotini (Fig. 4). No arteriovenous anastomoses have developed in any of the animals studied to date.

Retinal oedema was a frequent finding, and developed in the region of the arteriolar occlusion approximately three to 24 hours after operation. Initially the oedema appeared as a slight haze which was just visible when compared with the normal adjacent retina, but during the first week it became dense white and well delineated from the unaffected surrounding tissue. By the tenth to fourteenth day the whole process resolved leaving the affected areas with a rather atrophic appearance. It is interesting that some of the smaller patches of oedema closely resembled "cottonwool" spots (Fig. 5), and silver staining of one specimen revealed Cajal-type swellings of the nerve fibres in the area involved (Fig. $6 a$ and $b$ ). Three animals developed pre-retinal and intra-retinal haemorrhages in eyes with visible arteriolar ballotini, but not necessarily at the site of embolization (Fig. 7). Central cystic retinal changes including an instance of apparent "macular" cyst formation as well as "geographic" equatorial and peripheral atrophy of the retina were also observed (Figs 2 and 8). The former areas were closely related to the sites of ballotini occlusion, whereas the latter lesions occurred without obvious relation to retinal arteriolar block and may well have resulted from underlying choroidal involvement.

We have used a variety of experimental techniques to study the retinae and choroids of ballotini-treated animals. Indian ink injections have demonstrated most convincingly the various types of retinal vascular anastomoses and neovascularization which followed ballotini occlusion (Figs 4 and 9). Pepsin-trypsin digestions of the retina illustrated the cellular characteristics of the vascular anastomoses, and seemingly these channels, which develop within the existing capillary bed, assume the cellular characteristics of arterioles and venules (Fig. 10). Routine sections for light and electron microscopy are being prepared, but in both instances technical difficulties have arisen when cutting in the region of the ballotini.

\section{Discussion}

Attempts to produce embolization of the retinal arterioles in experimental animals date back to the late nineteenth century. Shiba (1906) states that Singer (1895), using an oil dye, was the first to produce emboli, and he was apparently able to demonstrate emboli in rabbits but not in dogs. Herrnheiser (1895), using Singer's method, produced retinal arterial emboli in both the rabbit and dog, and, in the former animal, claims to have demonstrated direct arteriovenous communications. In 1900 BirchHirschfeld injected pure mercury into the carotid artery of rabbits and was able to 


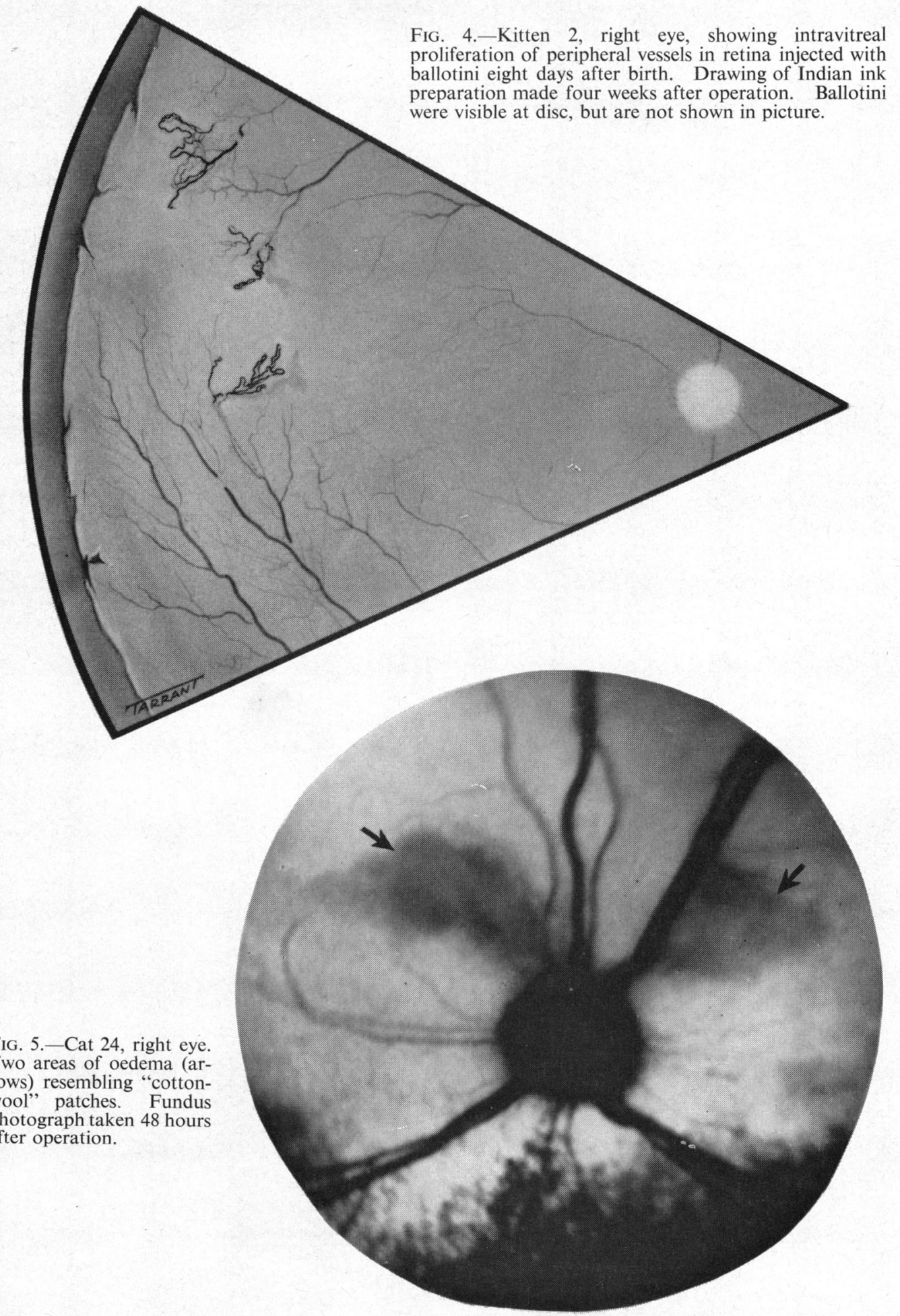




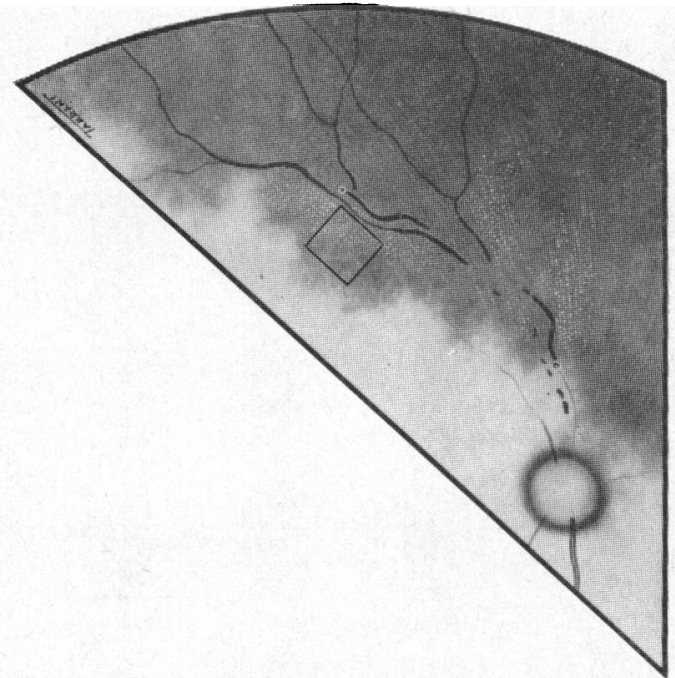

FIG. 6a.-Cat 12, left eye. Drawing of post-mortem appearance of swollen nerve fibres in areas surrounding intra-arterial ballotini, one week after operation.

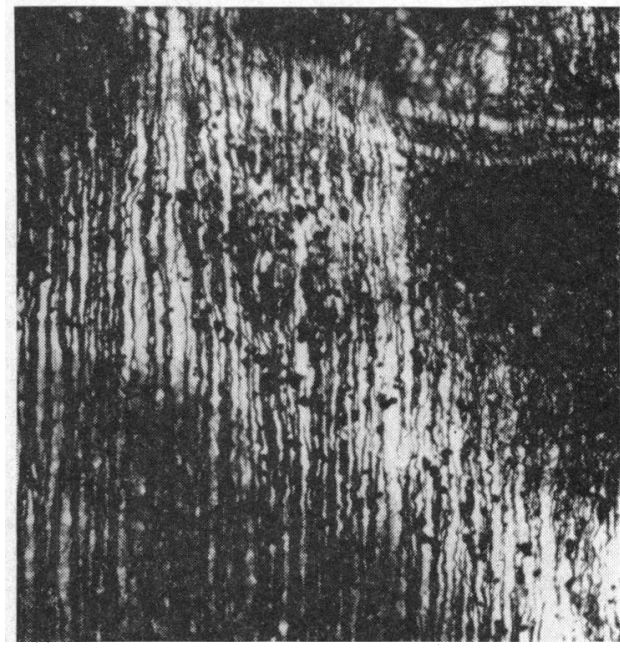

FIG. $6 b$.-Silver stained preparation of area of retina outlined by the square in Fig. $6 a$. Many nerve fibres show terminal nodular swellings. $\times 68$.

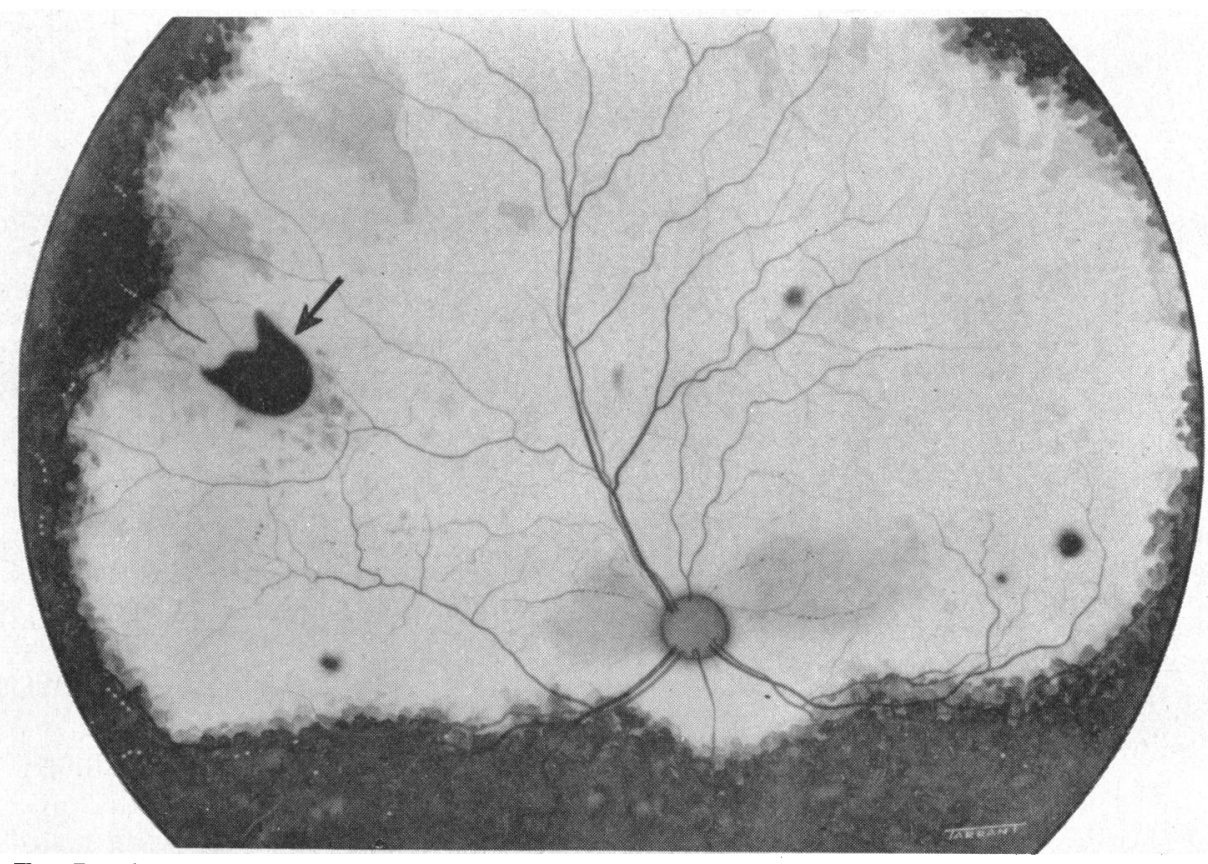

FIG. 7.-Cat 16, left eye. Pre-retinal (arrow) and multiple intra-retinal haemorrhages as seen two days after ballotini injection. Note localized areas of oedema on either side of optic disc.
(Fundus painting.) 


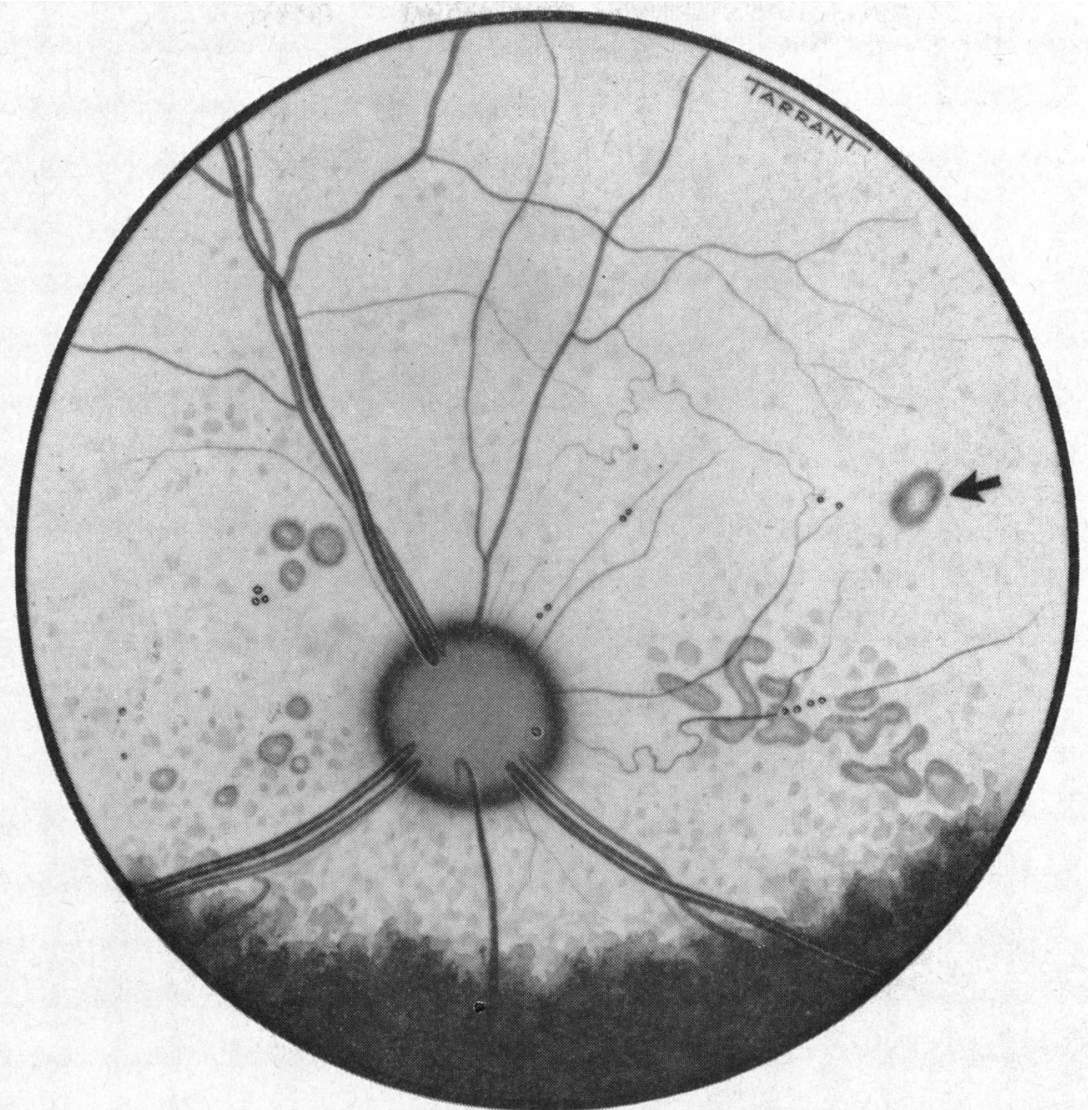

Fig. 8.-Cat 16, left eye. Multiple areas of cystic retinal atrophy on either side of disc eighteen days after operation. Round grey lesion (arrow) is a "macular" cyst. Note tortuous arterial anastomosis arising from disc margin at 4 o'clock. (Fundus painting.)

demonstrate mercury emboli. Shiba (1906) injected soot in liquid paraffin into the carotid artery of several rabbits, dogs, and cats. He terminated his experiments within 24 hours after injection and noted oedema in the nerve fibre and ganglion layers of the retina in four of eight animals. More recent attempts at retinal arteriolar embolization include those by Cook (1957), Hollenhorst, Lensink, and Whisnant (1962), Reinecke, Kuwabara, Cogan, and Weis (1962), and Hirose and Baba (1963). Cook (1957) studied the effect of experimental fat emboli in cats in relation to the pathogenesis of diabetic retinopathy, but described only localized areas of capillary dilatation and capillary closure in the retina. Hollenhorst and others (1962) injected a variety of agents including cholesterol, uric acid, atheromatous crystals, and pumicestone into the carotid arteries of several dogs and monkeys. These were essentially acute experiments, and those utilizing cholesterol produced bright intra-arterial plaques similar to those seen in the fundi of humans with carotid artery disease, while the experiments with uric acid and pumice-stone were less successful and provided little information. Reinecke and co-workers (1962) occluded isolated capillaries with fat emboli, but did not report clinical features of the occlusion, though they did note that retinal digest preparations showed acellular strands distal to the site of occlusion. 


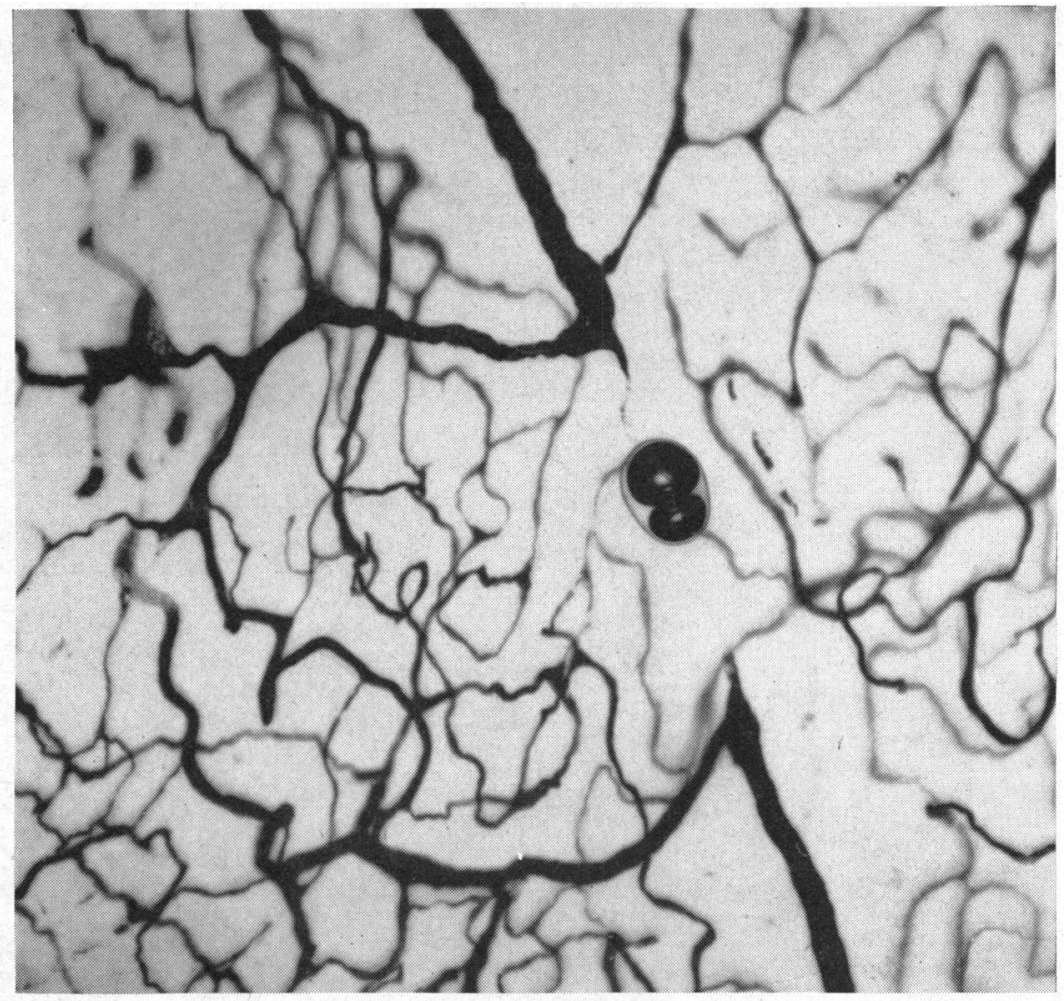

FIg. 9.-Cat 18, left eye. Retina injected with Indian ink showing a tortuous interarterial anastomosis linking the segments of an arteriole blocked by a glass ball, sixteen days after operation. $\times 165$.

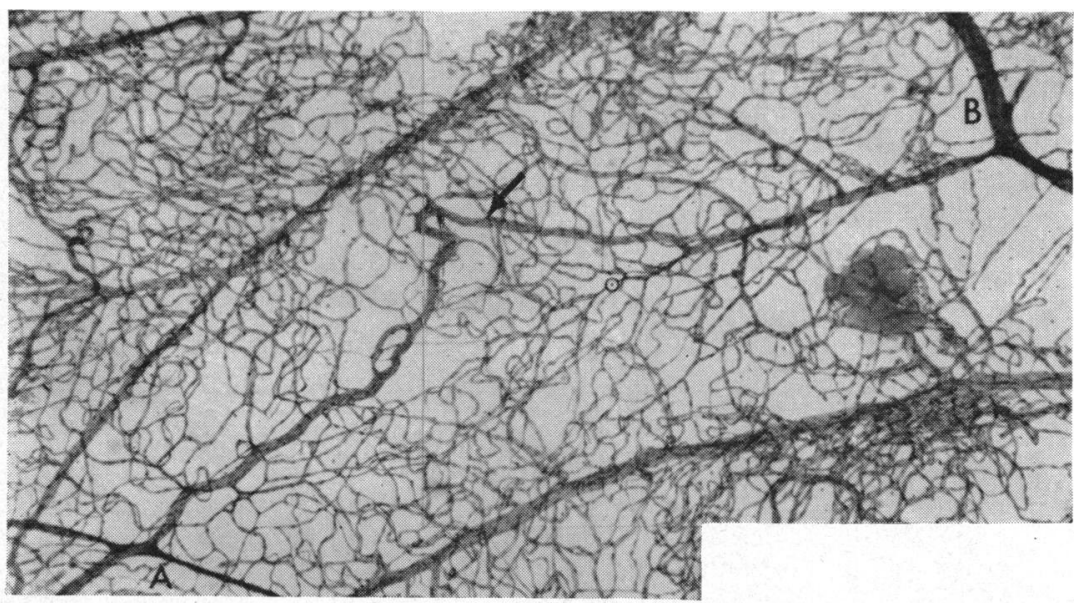

Fig. 10.-Cat 16, right eye. Pepsin-trypsin digest preparation showing the wellformed anastomosis (arrow) between blocked artery A and patent artery B, eighteen days after operation. $\times 79$. 
In an attempt to mimic "pulseless" disease, Hirose and Baba (1963) injected chopped foetal hair into the carotid arteries of rabbits and observed a variety of alterations in the retinal circulation. Among the effects mentioned were temporary arteriovenous anastomoses, increased visibility of the peripheral retinal vessels, delayed vascular obliteration, and temporary dilatation of the retinal arterioles and venules.

In our experiments the retinal haemorrhages, retinal oedema, and lesions resembling cotton-wool spots also have their counterparts in human disease, and it is especially instructive to have confirmed that cotton-wool spots can derive from arteriolar obstruction alone, which strengthens the concept that they may be primary foci of ischaemia rather than primary foci of capillary leakage-alternative hypotheses which have recently been debated by Ashton and Harry (1963) and Hodge and Dollery (1964).

We have also been impressed by the total absence of capillary micro-aneurysms in all our experiments, despite the formation of numerous interarteriolar and intervenular shunts through the capillary bed, which would seem to imply that micro-aneurysms cannot form in the animals employed-they have, however, been demonstrated in cats by Becker and Post (1951), and Mutlu and Leopold (1964)-or that these types of shunts through the capillary bed cannot alone produce aneurysms; this is a point of some relevance to the "shunt hypothesis" of diabetic retinopathy advanced by Cogan and Kuwabara (1963).

The production, simply by occluding the arterioles, of intravitreal vasoproliferation at the periphery of the immature retina of the kitten, exactly as occurs in retrolental fibroplasia, is a striking confirmation of the thesis that vasoproliferation in this disease is not directly concerned with oxygen toxicity per se but is merely the sequel of the obliteration of the vessels induced by oxygen (Ashton, 1957).

It seems likely, therefore, that the technique of occluding the retinal arterioles with ballotini will prove useful in the study of a number of retinal phenomena, both vascular and extravascular, especially in providing suitable material for electron microscopic studies.

The present work appears to be the first demonstration, both clinical and pathological, of the acute and chronic effects of inert, graded emboli upon the retinal vasculature of an experimental animal in which the retina resembles in many ways that of the human. Perhaps the most remarkable feature, apart from the vasoproliferation into the vitreous of the kitten, is the demonstration of the ability of the retinal vascular tree to form anastomotic communications, so by-passing occluded vessels and thereby probably limiting the resulting injury. These anastomoses appear to arise in existing capillary communications between arterioles and between venules and it is most interesting that arteriovenous communications do not develop.

Similar observations in man have been reported by several authors in cases of branch retinal artery occlusion; Jensen (1938) reviewed the literature, and Kornzweig, Eliasoph, and Feldstein (1964) have recently demonstrated arteriolar anastomoses in a retinal digest preparation of a patient with an old branch artery occlusion. We have not yet determined whether these channels do, in fact, develop rapidly enough to prevent the surrounding retinal tissue from dying, a point most important from the clinical aspect. 
We should like to thank Mrs. Patricia Rawlings and Mr. George Knight for technical assistance, Miss E. FitzGerald for secretarial help, and Mr. T. Tarrant of the Medical Illustration Department for providing the fundus paintings. We are grateful to Professor F. Vrabec for preparing the silver stained specimen.

\section{REFERENCES}

Ashton, N. (1957). Amer. J. Ophthal., 44, October, Pt 2, 7. and HARRY, J. (1963). Trans. ophthal. Soc. U.K., 83, 91.

BeCKer, B., and Post, L. T., Jr. (1951). Amer. J. Ophthal., 34, 677.

Birch-Hirschfeld, A. (1900). Albrecht v. Graefes Arch. Ophthal., 50, 166.

Cogan, D. G., and Kuwabara, T. (1963). Diabetes, 12, 293.

CoOK, C. A. G. (1957). Brit. J. Ophthal., 41, 741.

Herrnheiser, J. (1895). Klin. Mbl. Augenheilk., 33, 315.

Hirose, K., and BABA, K. (1963). Amer. J. Ophthal., 55, 554.

Hodge, J. V., and Dollery, C. T. (1964). Quart. J. Med., 33, 117.

Hollenhorst, R. W., Lensink, E. R., and Whisnant, J. P. (1962). Trans. Amer. ophthal. Soc., $60,316$. JENSEN, V. A. (1938). Acta ophthal. (Kbh.), 16, 485.

Kornzweig, A. L., EliasopH, I., and Feldstein, M. (1964). Arch. Ophthal. (Chicago), $71,542$.

MutLU, F., and LeOPOLD, I. H. (1964). Ibid., 72, 513.

Reinecke, R. D., Kuwabara, T., Cogan, D. G., and Weis, D. R. (1962). Ibid., 67, 470.

Shiba, S. (1906). Albrecht v. Graefes Arch. Ophthal., 63, 393.

SINGER (1895). Quoted by Herrnheiser (1895) and Shiba (1906). 